\title{
Adaptive Turbulent Schmidt Number Approach for Multi- Scale Simulation of Supersonic Crossflow
}

\author{
Ez Hassan $^{*}$ and Hikaru Aono ${ }^{\dagger}$ \\ University of Michigan, Ann Arbor, MI \\ John Boles \\ Taitech, Inc., Beavercreek, $\mathrm{OH}$ \\ Douglas Davis ${ }^{\S}$ \\ Air Force Research Laboratory, Fairborn, $\mathrm{OH}$ \\ Wei Shyy ${ }^{* * \dagger}$ \\ University of Michigan, Ann Arbor, MI \\ The Hong Kong University of Science and Technology, Kowloon, Hong Kong
}

\begin{abstract}
The multi-scale turbulence approach is useful in predicting mean flows in problems containing complex turbulent structures that are otherwise unattainable using standard Reynolds-averaged Navier-Stokes models. In crossflow simulations using the multi-scale turbulence approach, turbulent mass diffusion in the resolved field showed variations not correlated with the eddy viscosity. This work is aimed at modifying the multi-scale turbulence approach to allow the resolved field to adaptively influence the value of turbulent Schmidt number in the Reynoldsaveraged Navier-Stokes sub-filter model. The proposed model estimates a time-averaged resolved turbulent Schmidt number that is used in place of the constant value common to standard Reynolds-averaged Navier-Stokes approaches. This approach is assessed by grid refinement study in which different amounts of turbulence are resolved. Fuel concentration predictions show an improvement when compared with experimental measurements versus the multi-scale model without the adaptive approach.
\end{abstract}

$\begin{array}{ll} & \\ C_{p} & =\text { heat capacity at constant pressure } \\ C_{g s} & =\text { generalized coefficient for numerical viscosity estimation } \\ C_{s} & =\text { Smagorinsky constant } \\ D & =\text { injector inner diameter }\end{array}$

\footnotetext{
* PhD Candidate, Aerospace Engineering Department

${ }^{\dagger}$ Postdoctoral Research Fellow, Aerospace Engineering Department

* Research Scientist, AFRL/RZAS

$\S$ Aerospace Engineer, AFRL/RZAS

** Clarence L. "Kelly" Johnson Collegiate Professor, Aerospace Engineering Department

${ }^{\dagger}$ Chair Professor, Mechanical Engineering at HKUST
}

American Institute of Aeronautics and Astronautics 


\begin{tabular}{|c|c|}
\hline$D_{i_{m}}$ & $=$ diffusion vector for species $m$ \\
\hline E & $=$ total specific energy of the mixture \\
\hline$H$ & $=$ total specific enthalpy of the mixture \\
\hline$F_{2}$ & $=$ second blending function, Menter SST \\
\hline$f_{d}$ & $=$ filter function \\
\hline$h_{m}$ & $=$ species specific enthalpy \\
\hline$k$ & $=$ turbulent specific kinetic energy \\
\hline$L_{G}$ & $=$ length scale based on cell size \\
\hline$L_{T}$ & $=$ measure of turbulence length scale \\
\hline NS & $=$ total number of species \\
\hline$p$ & $=$ pressure \\
\hline$P r_{t}$ & $=$ turbulent Prandtl number \\
\hline$q_{i}$ & $=$ heat transfer vector \\
\hline$S_{i j}(\tilde{u})$ & $=$ strain rate tensor of Favre-averaged subgrid RANS solution \\
\hline$S c_{t}$ & $=$ turbulent Schmidt number \\
\hline$T$ & $=$ mixture temperature \\
\hline$u_{i}$ & $=$ mixture velocity \\
\hline$Y_{m}$ & $=$ mole fraction of species $\mathrm{m}$ \\
\hline$\mu_{t}$ & $=$ dynamic eddy viscosity \\
\hline$\mu_{\text {num }}$ & $=$ dynamic numerical viscosity \\
\hline$\underline{\underline{t}}$ & $=$ kinematic eddy viscosity \\
\hline$\overline{\dot{\omega}}$ & $=$ average chemistry source term \\
\hline$\rho$ & $=$ mixture density \\
\hline$\tau_{i j}$ & $=$ stress tensor \\
\hline$(\sim)$ & $=$ Favre average operator \\
\hline$(-)$ & $=$ time average operator \\
\hline $\mathrm{R}$ & $=$ superscript for Reynolds terms \\
\hline Res & $=$ subscript for quantities calculated from resolved field \\
\hline $\mathrm{R}$ & $\begin{array}{l}=\text { superscript for fluctuations in the sub-filter portion of the field } \\
=\text { superscript for fluctuations in the resolved portion of the field }\end{array}$ \\
\hline
\end{tabular}

\section{Introduction}

CUPERSONIC crossflow analysis is needed to understand the physics behind supersonic combustion occurring in scramjet engines. Because the residence time of the flow in the combustor is often on the order of chemical time scales, it is of utmost importance for the fuel and oxidizer to be mixed quickly. It is necessary to gain better understanding of the effect of different fuel injection configuration and combustor geometries on the mixing process in order to achieve desirable designs for scramjet engines.

Reynolds-averaged Navier-Stokes (RANS) based approaches have shown some success in understanding the mechanics of supersonic cross flows. Tam et al. ${ }^{1}$ used RANS based methods with Menter Shear Stress Transport $\left(\mathrm{SST}^{2}\right)$, Menter baseline $\left(\mathrm{BSL}^{2}\right)$, and Wilcox $k-\omega^{3}$ turbulence models to simulate the experiments of Gruber et al. ${ }^{4-6}$. It was shown that the Wilcox $k-\omega$ model performed the best of all three; however, in all the models tested the fuel penetration height was over predicted by up to $25 \%$. Palekar et al. ${ }^{7}$ obtained better correlations with penetration heights with a 4.7 million cell grid using the commercial computational fluid dynamics (CFD) code GASP. The Wilcox $k-\omega$ turbulence model was also utilized for turbulence closure. Coarser grids did not sufficiently resolve the flow and spanwise fuel penetration significantly deviated from the experiment. Maddalena et al. ${ }^{8}$ used the $k-\omega$ Wilcox turbulence model to simulate an aeroramp injection scheme as well as transverse injection of sonic helium into air. Total pressure loss for transverse injection was shown to be greater than of normal injection configuration with the computational results not correlating well with the experiment.

As stated by many researchers ${ }^{9-11}$, there are some obvious limitations to RANS models when applied to unsteady problems because they tend to be overly dissipative (predicting higher eddy viscosity and damping the unsteady motion of the fluid). The crossflow problem usually involves large scale unsteady turbulent structures, density gradients, and shock boundary layer interactions as evident by experimental findings ${ }^{4-6}$. These phenomena are 
difficult to capture correctly with standard RANS approaches and may benefit from methods capable of resolving turbulent structures such as Direct Numerical Simulation (DNS) or Large Eddy Simulation (LES). LES is capable of capturing the large scale turbulent structures, and while not as computationally expensive as DNS, it is impractical for use in the supersonic crossflow problem because a prohibitively large number of grid points must be used to resolve the boundary layer. Recently, Kawai and Lele ${ }^{12}$ conducted a LES of sonic injection into a supersonic crossflow and showed key physics of the jet mixing in supersonic crossflow such as clockwise and counterclockwise rotating strong counter-rotating vortices, a pair of U-shaped counter rotating vortices. To reduce the expense of the computations, the Reynolds number was lowered by a factor of six, relative to the experiment ${ }^{13}$ but the boundary layer thickness upstream of jet injection was matched.

Detached eddy simulation (DES) ${ }^{11}$ and hybrid RANS/LES methods, where RANS is used at the wall boundary and LES is used elsewhere, has shown a great promise in the solution of supersonic crossflow problem.

Peterson et al. ${ }^{14}$ used a DES model based on Spalart-Allmaras one equation turbulence model to simulate supersonic cross flow experiments conducted at Virginia Polytechnic University. DES compared favorably to the experiment with results superior to RANS. Boles et al. ${ }^{15-17}$ simulated Gruber et al. ${ }^{4-6}$ air and helium injection cases as well as ethylene injection case conducted at Air Force Research Laboratory (AFRL) and reported by Lin et al. ${ }^{18}$. In all cases time averaged hybrid results were superior to RANS quantitatively and qualitatively when compared to experimental injectant distribution.

In an effort similar to hybrid RANS/LES, Hassan et al. ${ }^{19}$ used the multi-scale turbulence approach to reduce the eddy viscosity in SST turbulence model ${ }^{2}$. They were able to capture large scale turbulent structures showing remarkable improvement in the simulation of Lin et al. ${ }^{18}$ over the RANS approach. Grid refinement studies on adapted unstructured grids showed that very fine grids are needed to resolve enough turbulent scales to reach any form of grid independence. This was probably due to the use of the dissipative second-order upwind schemes. It was also shown that RANS simulations are very sensitive to the turbulent Schmidt number $\left(S c_{t}\right)^{19-20}$. Hassan et al. ${ }^{19}$ and Boles et al. ${ }^{17}$ derived estimates for the turbulent Schmidt number based on the resolved field which was not constant with strong variations throughout the flow.

There have been many efforts in the RANS community to calculate, rather than specify, the turbulent Prandtl and Schmidt numbers as early as $1975^{21}$. Methods based on the mixing length used a two equation model to calculate turbulent diffusivity in conjunction with $k-\varepsilon^{22-23}$. In general, the results from these methods showed an improvement over $k-\varepsilon$ alone in low temperature high Mach number cases. Guo et al. ${ }^{24}$ used a genetic algorithm to obtain model constants for a diffusion vector transport equation used in addition to $k-\varepsilon$. The results showed some improvement over a baseline $k-\varepsilon$ model for a jet-in-crossflow application. CRAFT Tech developed a variable Prandtl $\left(P r_{t}\right)$ and $\operatorname{Schmidt}\left(S c_{t}\right)$ number approach based largely on earlier efforts ${ }^{22-23}$ with added compressibility correction. The model showed improvements over a constant $P r_{t} / S c_{t}$ in a range of classical validation cases ${ }^{25-26}$.

Keistler $^{27}$ used a reacting model with variable $P r_{t} / S c_{t}$ method designed for high speed flows and based on CRAFT tech efforts ${ }^{25-26}$ to simulate Lin et al ${ }^{18}$ mixing case. The results were compared to those obtained by hybrid RANS/LES method of Boles et al. ${ }^{17}$ While the variables approach showed some limited improvement over RANS in predicting fuel concentration levels. It fell short to the hybrid RANS/LES method in predicting jet shape mainly due to inability to produce large scale turbulent structures.

To the authors' knowledge, there have been limited or no efforts in the literature to combine both hybrid RANS/LES type approaches with variable turbulent Schmidt number methods. In this effort we propose an extension to the multi-scale approach of Hassan et al. ${ }^{19}$ allowing for the calculation of the turbulent Schmidt number. The proposed method does not utilize transport equations with ad hoc constants. The value of the turbulent Schmidt number in the sub-filter RANS model is adaptively changed based on the resolved turbulent field. At every time step, the average turbulent Schmidt number based on the ratio of the resolved mass and momentum eddy viscosity is calculated. This value is used in the mass transport equation instead of the specified constant value. The method assumes that $S c_{t}$ is the same in all turbulent scales, and in this effort we evaluate this assumption with the Lin et al. ${ }^{19}$ mixing case.

The test case considered in this effort is based on experiments conducted at AFRL and reported by Lin et al. ${ }^{18}$ for injection of sonic ethylene into Mach 2 air. Injection was conducted at either 90 or 30 degrees with momentum ratios varying from 0.25 to 1.5 . We focus here on the normal injection with momentum ratio of 0.5 . We conduct the simulations on two different grids and compare to the previous results without the variable approach, RANS, and experimental measurements. We also present the results of the model while incorporating the effect of numerical viscosity. 


\section{Governing Equations and Computational Modeling Approaches}

A density-based, finite volume code, Loci-Chem ${ }^{28-29}$, is utilized in this study. The code is capable of handling mixed element type unstructured grids. The convective fluxes are based on Roe's flux difference splitting ${ }^{30}$. Both convective and diffusive fluxes are evaluated to second order accuracy. Menter SST ${ }^{2}$ model is used for turbulence closure along with multi-scale treatment.

\section{Multi-Scale Turbulence Treatment:}

With the concept of eddy viscosity used in either a standard two-equation RANS approach or the Smagorinsky subgrid model in LES, both filtered and averaged mass, momentum, and energy equations yield an identical mathematical form as indicated by Germano ${ }^{31}$,

$$
\begin{gathered}
\frac{\partial}{\partial t}\left(\bar{\rho} \tilde{Y}_{m}\right)+\frac{\partial}{\partial x_{i}}\left(\bar{\rho} \tilde{Y}_{m} \tilde{u}_{i}\right)=\frac{\partial}{\partial x_{i}}\left(D_{i_{m}}+D_{i_{m}}^{R}\right)+\bar{\omega} \\
\frac{\partial}{\partial t}\left(\bar{\rho} \tilde{u}_{i}\right)+\frac{\partial}{\partial x_{j}}\left(\bar{\rho} \tilde{u}_{i} \tilde{u}_{j}\right)=-\frac{\partial \bar{p}}{\partial x_{j}}+\frac{\partial}{\partial x_{j}}\left(\tau_{i j}+\tau_{i j}^{R}\right) \\
\frac{\partial}{\partial t}(\bar{\rho} \tilde{E})+\frac{\partial}{\partial x_{j}}\left(\bar{\rho} \widetilde{H} \tilde{u}_{j}\right)=\frac{\partial}{\partial x_{j}}\left[\tilde{u}_{j}\left(\tau_{i j}+\tau_{i j}^{R}\right)-\left(q_{j}+q_{j}^{R}\right)\right]
\end{gathered}
$$

where the Reynolds terms are modeled as follows:

$$
\begin{gathered}
D_{i_{m}}^{R} \approx-\frac{\mu_{t}}{S c_{t}} \frac{\partial \tilde{Y}_{m}}{\partial x_{j}} \\
\tau_{i j}^{R} \approx \mu_{t}\left(\frac{\partial \tilde{u}_{i}}{\partial x_{j}}+\frac{\partial \tilde{u}_{j}}{\partial x_{i}}-\frac{2}{3} \frac{\partial \tilde{u}_{k}}{\partial x_{k}} \delta_{i j}\right) \\
q_{j}^{R} \approx-\frac{\mu_{t}}{P r_{t}} \frac{\partial \tilde{T}}{\partial x_{j}}-\sum_{m=1}^{N S} h_{m} \frac{\mu_{t}}{S c_{t}} \frac{\partial \tilde{Y}_{m}}{\partial x_{j}}
\end{gathered}
$$

The variables in Eqs. (1) and (2) are shown in the filtered form, however they could represent averaged quantities in which case the value of the eddy viscosity would be produced via a two equation turbulence model. They could also be instantaneous variables when the eddy viscosity is set to zero. When they are filtered, a Smagorinsky subgrid eddy viscosity is defined as follows:

$$
\begin{gathered}
\mu_{t}=\bar{\rho} C_{s} L_{G}^{2} S \\
S=\sqrt{\frac{\partial \tilde{u}_{i}}{\partial x_{j}} \frac{\partial \tilde{u}_{j}}{\partial x_{i}}+\frac{\partial \tilde{u}_{i}}{\partial x_{j}} \frac{\partial \tilde{u}_{i}}{\partial x_{j}}-\frac{2}{3}\left(\frac{\partial \tilde{u}_{i}}{\partial x_{i}}\right)^{2}}
\end{gathered}
$$


Assuming that we average the result of each model, Eqs. (1) and (2) combined with the averaging procedure, become a single mathematical formulation in which we input an eddy viscosity and output averaged quantities. This formulation is valid for averaged, filtered and instantaneous equations as long as the correct eddy viscosity value is provided.

In multi-scale modeling, we assume the input eddy viscosity is a continuous function that varies from the RANS value to zero depending on the ratio of the grid size, $L_{G}$, to a locally defined turbulence length scale, $L_{T}$. After the appropriate eddy viscosity is found it is used in Eqs. (1) and (2). The multi-scale model allows smooth transition from RANS to LES to DNS with the grid size and local turbulent length scales being the determining factors of which model to use. When employing this approach, there are no limitations on grid size or geometry because the transition between the models is allowed to occur anywhere in the computational domain.

The multi-scale treatment can be implemented easily into any RANS code as a mere modification to the eddy viscosity that is output from the two equation model, before using it in the mass, momentum and energy transport. This modification depends on the definition of the filter function, the turbulence length scale $\left(L_{T}\right)$ and the multiscale eddy viscosity.

\section{Multi-scale approach}

A multi-scale turbulence approach proposed by Hassan et al. ${ }^{19}$ is used in this effort. It is based on a filter function that is a blend between the filter-based model (FBM) developed by Johansen et al. ${ }^{32}$ and the multi-scale hybrid RANS/LES turbulence model developed by Nichols and Nelson ${ }^{33}$. The turbulence length scale, $L_{T}$, is defined as follows:

$$
L_{T}=\frac{\sqrt{k}}{C_{4} \max \left(\omega, S F_{2} / a_{1}\right)}
$$

where,

$$
C_{4}=\frac{C_{s}}{\sqrt{0.3}}
$$

and the eddy viscosity defined as,

$$
v_{t}=v_{t_{R A N S}} f_{d}
$$

The constant, $a_{1}$, is the same one used in SST model ${ }^{2}$. The value of $C_{s}$ varies, but in this approach we use the value of 0.01 as recommended by Boles ${ }^{17}$ for ethylene injection cases. The grid length scale length scale, $L_{G}$, is defined as twice the maximum distance between the cell center and each face as follow

$$
L_{G}=2 \max \left|\vec{X}_{\text {center }}-\vec{X}_{\text {face }}\right|
$$

Finally the filter function takes the form adopted in Hassan et al. ${ }^{19}$,

$$
f_{d}=\frac{1}{2}\left\{1+\tanh \left[2 \pi\left(\frac{1}{1+\left(\frac{L_{T}}{2 L_{G}}\right)^{4 / 3}}-0.5\right)\right]\right\}+0.0622
$$




\section{Adaptive turbulent Schmidt number approach}

In standard RANS approach the turbulent momentum flux is modeled as follows,

$$
\begin{gathered}
-\overline{\rho u_{\imath}^{\prime} u_{\jmath}^{\prime}}=\mu_{t} S_{i j}\left(\tilde{u}_{i}\right)-\frac{2}{3} \overline{\rho k} \delta_{i j} \\
S_{i j}\left(\tilde{u}_{i}\right)=\left(\frac{\partial \tilde{u}_{i}}{\partial x_{j}}+\frac{\partial \tilde{u}_{j}}{\partial x_{i}}-\frac{2}{3} \frac{\partial \tilde{u}_{k}}{\partial x_{k}} \delta_{i j}\right)
\end{gathered}
$$

Turbulent mass flux for multispecies is modeled as,

$$
-\overline{\rho u_{\imath}^{\prime} Y_{m}^{\prime}}=\frac{\mu_{t}}{S c_{t}} \frac{\partial \tilde{Y}_{m}}{\partial x_{i}}
$$

We can multiply Eq. (9) by $S_{i j}\left(\tilde{u}_{i}\right)$ and Eq. (10) by $\partial \tilde{Y}_{m} / \partial x_{i}$ to obtain scalar equations. Then we can define mass and momentum eddy viscosities based on turbulent fluctuations,

$$
\begin{gathered}
v_{t, m o m}=\frac{\mu_{t}}{\bar{\rho}}=-\frac{\left(\overline{\rho u_{\imath}^{\prime} u_{\jmath}^{\prime}}-\frac{2}{3} \overline{\rho k} \delta_{i j}\right) S_{i j}\left(\tilde{u}_{i}\right)}{\bar{\rho} S_{i j}\left(\tilde{u}_{i}\right) S_{i j}\left(\tilde{u}_{i}\right)} \\
v_{t, \text { mass }}=\frac{\mu_{t}}{S c_{t} \bar{\rho}}=-\frac{\left(\overline{\rho u_{l}^{\prime} Y_{m}^{\prime}}\right)}{\bar{\rho}} \frac{\left(\partial \tilde{Y}_{m} / \partial x_{i}\right)}{\left(\partial \tilde{Y}_{m} / \partial x_{i}\right)\left(\partial \tilde{Y}_{m} / \partial x_{i}\right)}
\end{gathered}
$$

The turbulent Schmidt number can be obtained by dividing the mass and momentum eddy viscosities. When using the multi-scale model approach only fluctuations in the resolved field are calculated directly, while those in the sub-filter field are calculated using the RANS model. To eliminate uncertainties associated with specifications of the turbulent Schmidt number. We defined the resolved turbulent Schmidt number based on resolved quantities as follows,

$$
S c_{t_{-} R e s}=\frac{\frac{\left(\overline{\rho u_{l}^{R} u_{J}^{R}}-\frac{2}{3} \overline{\rho k_{R e s}} \delta_{i j}\right) S_{i j}\left(\tilde{u}_{i}\right)}{\bar{\rho} S_{i j}\left(\tilde{u}_{i}\right) S_{i j}\left(\tilde{u}_{i}\right)}}{\frac{\left(\overline{\rho u_{l}^{R} Y_{m}^{R}}\right)}{\bar{\rho}} \frac{\left(\partial \tilde{Y}_{m} / \partial x_{i}\right)}{\left(\partial \tilde{Y}_{m} / \partial x_{i}\right)\left(\partial \tilde{Y}_{m} / \partial x_{i}\right)}}
$$

The use of the resolved turbulent Schmidt number in the RANS model assumes that the ratio of turbulent momentum fluxes and turbulent mass fluxes in the resolved portion of the flow is the same as in the unresolved portions of the flow. In other words if we split the energy spectrum at a certain wave length equal to the filter function we have equal ratios of mass and momentum turbulent fluxes in the sub-filter and resolved portions. While this seems like a very reasonable assumption, it does require further investigation. The results and cases presented in this paper could be regarded as validation for this assumption. 
The resolved turbulent Schmidt number $\left(S c_{t_{-} \text {Res }}\right)$ is calculated at every time step during the simulation and is used in the RANS mass transport equation. The value of the resolved turbulent Schmidt number is theoretically constant at every time step however, because averages are not available a priori, the estimations of the averages improve as the simulation proceeds and $S c_{t_{-} R e s}$ converge to a constant value. Also the application of $S c_{t_{-} R e s}$ into the RANS sub-filter model is not done until a number of iterations at constant $S c_{t}$ to avoid erroneous values of $S c_{t_{-} R e s}$ at the beginning of the simulation. Also the positivity of the resolved turbulent Schmidt number is maintained by taking the absolute value.

Lin et al. ${ }^{18}$ performed a sonic injection of ethylene into Mach 2 air crossflow at the continuous flow supersonic tunnel at Wright-Patterson Air Force Base, Ohio. The tunnel has a constant test area of $131 \times 152 \mathrm{~mm}$. The injectors were circular with different diameters, injection angles, and momentum ratios. In this study we focus on the case with $D=0.1875$ inches normal injection with a momentum ratio of 0.5 . Lin et al. ${ }^{18}$ used Raman scattering technique to collect quantitative concentration data. These measurements were used to calculate the mixture fraction at various $x / D$ locations downstream of the injector.

\section{Grid and numerical choices}

In our previous study ${ }^{19}$, three different grids were used with mixed structured domains as shown in Figure 1 and Figure 2. In this study, however, only the coarse and intermediate grids are used in this effort and will be named grid 1 , and grid 2 respectively. Grid 1 contains about 7 million cells with the jet region at a finer resolution and a boundary layer grid starting at $\boldsymbol{y}^{+} \sim 10$. Grid 2 was adapted from the grid 1 based on the filter function and ethylene mole fraction and contains about 17 million cells.

The inlet to the computational domain was taken from a RANS solution of a separate simulation of the nozzle. The full width and height of the wind tunnel was used in the computation. The injector geometry was also representative of that used in the experiment. Boundary conditions and geometrical information of the simulation are shown in Table 1.

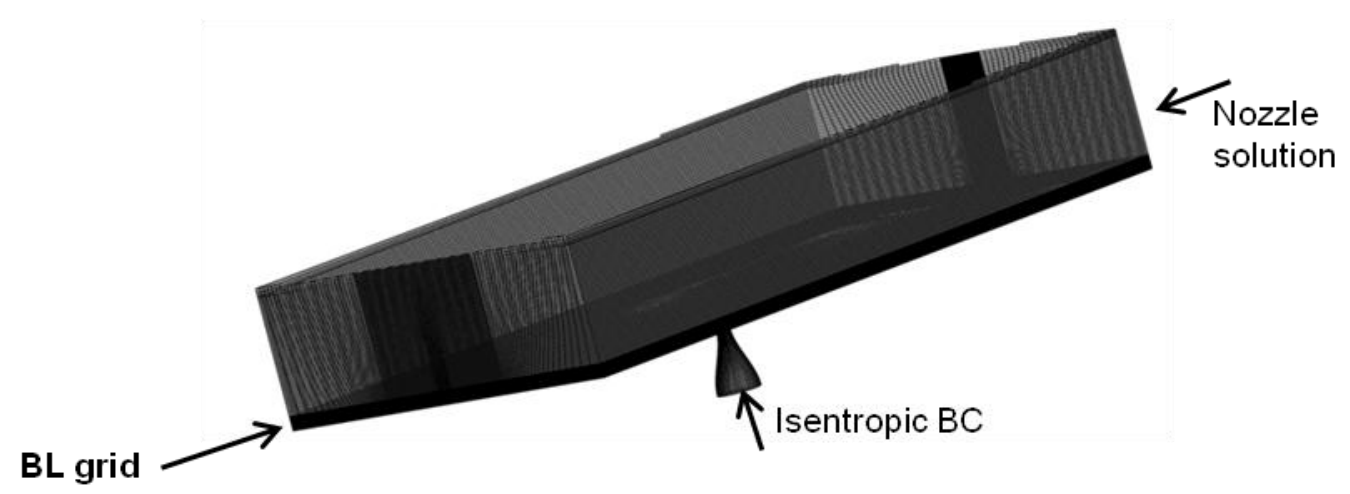

Figure 1. Grid 1 cell distributions for multi-scale simulation.
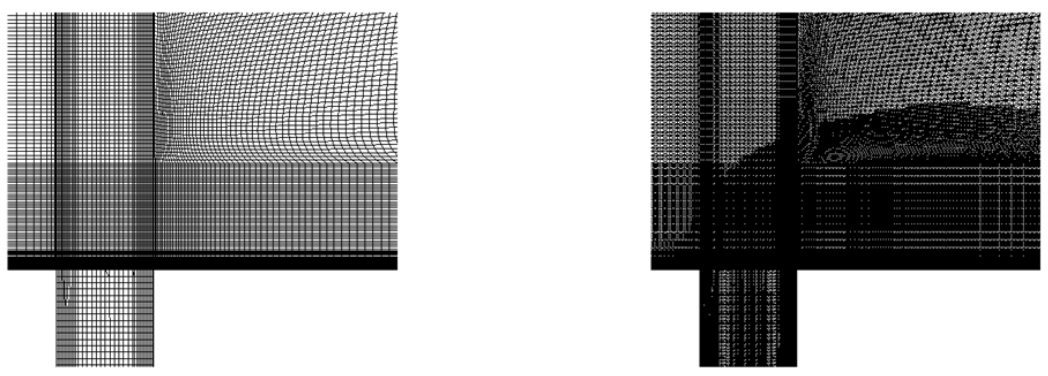

Figure 2. Center plane cut. Grid1 (left) and grid 2 (right). 
Table 1. Flow and geometric conditions for Ethylene injection.

\begin{tabular}{c|c}
\hline Parameters & Values \\
\hline$P_{0}$ (freestream) & $244 \mathrm{KPa}$ \\
$T_{0}$ (freestream) & $300 \mathrm{~K}$ \\
$M$ (freestream) & 2 \\
$P_{0}$ (injectant) & $127.5 \mathrm{KPa}$ \\
$T_{0}$ (injectant) & $322 \mathrm{~K}$ \\
$D$ & $4.8 \mathrm{~mm}$ \\
$q$ & 0.5 \\
Injectant angle $(\theta)$ & $90^{\circ}$ \\
\hline
\end{tabular}

The cases used in this study are a combination of those done previously without the adaptive $\boldsymbol{S} \boldsymbol{c}_{\boldsymbol{t}}$ approach and those done with the new approach and others that will be defined in further discussion. Table 2 gives a summary of the cases and their description to facilitate reference to them in the results section.

Table 2. Description of simulation cases discussed in this effort.

\begin{tabular}{c|cccc}
\hline Case & $\begin{array}{c}\text { Grid } \\
\text { resolution }\end{array}$ & $\begin{array}{c}\text { Turbulence } \\
\text { treatment }\end{array}$ & $S c_{t}$ & $\begin{array}{c}\mu_{\text {num }} \\
\text { correction }\end{array}$ \\
\hline $\begin{array}{c}\text { Previous } \\
1\end{array}$ & $600 \mathrm{~K}$ & RANS-SST & 0.7 & No \\
2 & Grid $1 / 7000 \mathrm{~K}$ & Multi-scale-SST & 0.7 & No \\
3 & Grid 2/17000K & Multi-scale-SST & 0.7 & No \\
New & & Multi-scale-SST & Adaptive & No \\
4 & Grid 1/ 7000K & Multi-scale-SST & Adaptive & No \\
5 & Grid 2/17000K & Multi-scale-SST & Adaptive & Yes \\
6 & Grid 1/ 7000K & Multi-scale-SST & Adaptive & Yes \\
7 & Grid 2/17000K & MILES (no model) & N/A & N/A \\
8 & Grid 1/ 7000K & M & &
\end{tabular}

\section{Results and Discussion}

The simulations were run with the SST turbulence model $^{2}$ until converged to a RANS solution. The multi-scale approach with the adaptive turbulent Schmidt number was then applied and instantaneous results were collected and averaged. Results with the adaptive $S c_{t}$ approach are compare to experimental, RANS, and standard multi-scale results. The simulations were run on grids 1 and 2 . A numerical viscosity correction for the adaptive $S c_{t}$ approach is discussed and results are presented and compared. Consequently, the mixing process is discussed in terms of the fluid dynamics. Furthermore, pressures on the bottom and center plane for the adaptive $S c_{t}$ approach and standard multi-scale are compared to validate the integrity of the adaptive $S c_{t}$ approach.

\section{A. Adaptive $S c_{t}$ multi-scale and comparison to constant $S c_{t}$ multi-scale and RANS results}

The adaptive $S c_{t}$ multi-scale approach explained in earlier section is implemented and tested with both grid 1 and grid 2 shown in Fig. 2. To be consistent, it is compared to RANS solution with $S c_{t}=0.7$ and earlier multi-scale solutions with $S c_{t}=0.7^{19}$. It should be noted that in an earlier study RANS results were very sensitive to $S c_{t}$ and 
comparable levels of fuel mass fractions were obtained by drastically lowering its value ${ }^{19}$. When running the simulation a constant $S c_{t}$ of 0.7 is used for the first 2000 iterations while an average is collected for the adaptive resolved $S c_{t}$. With numerical experiments, the resolved $S c_{t}$ was independent of the initial constant value used in the first 2000 iterations.

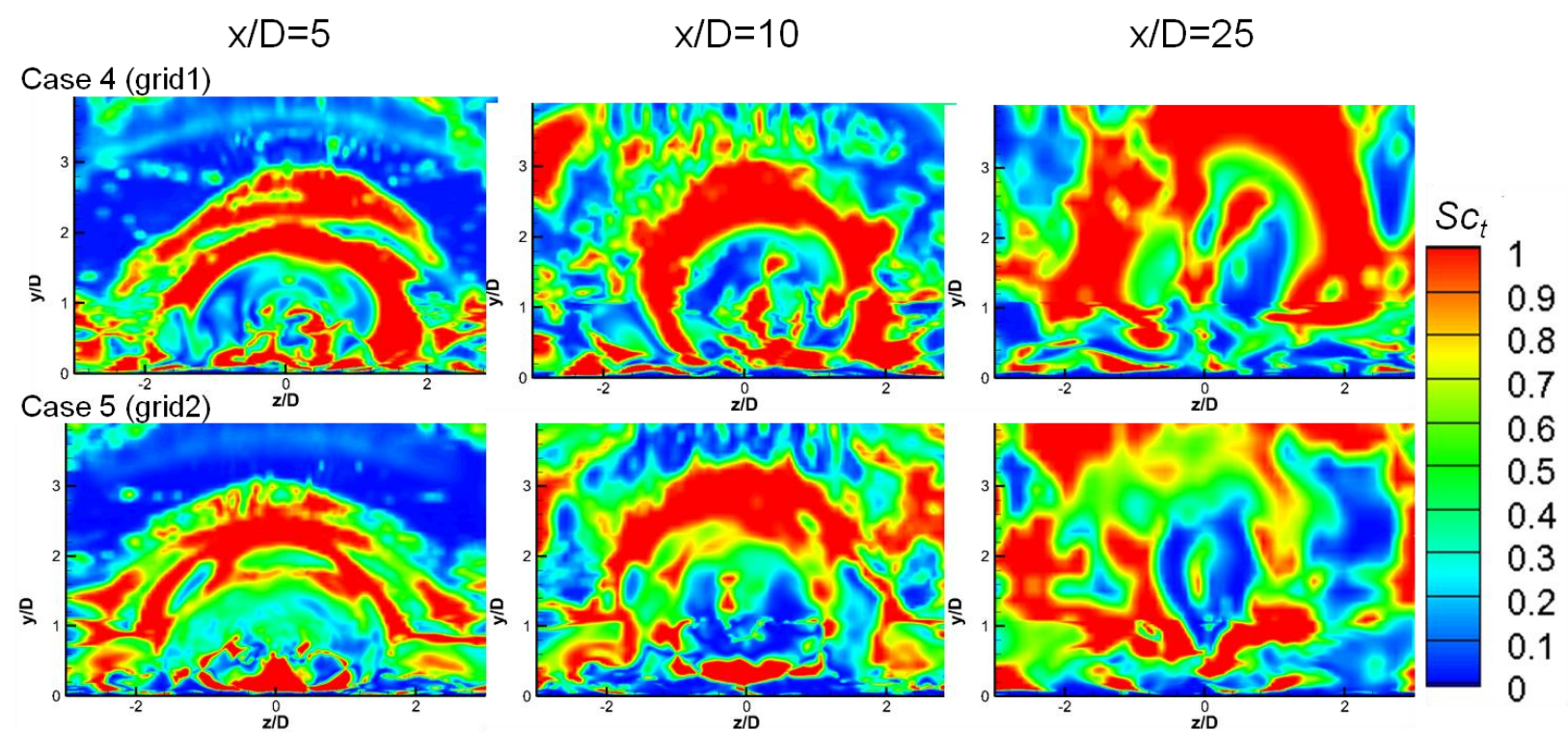

Figure 3. Adaptive turbulent Schmidt number contours for grid 1(top) and 2 (bottom) at different $x / D$ locations.

The resolved turbulent Schmidt number contours for grid 1 and grid 2 are shown in Figure 3 .Those contours are an indication of the mixing in the resolved field of each grid. The range is limited from 0 to 1 because larger values imply negligible turbulent mass diffusivity. Also these values are irrelevant in areas that do not contain fuel. Overall features of the distribution of the turbulent Schmidt number are similar in grid 1 and grid 2. Distributions in grid 1 however, are not fully symmetric, and symmetry improves when the grid is refined. This shows that for grid 2, the resolved field is strong enough to overcome errors induced by approximate averaging procedures. Symmetry and similarity between the two grids are better in the near field region where the grid is finer than the rest of the domain. In the far field where the grid is coarser and less resolved mixing occurs, the results seem to be less symmetric and more differences are observed between grid 1 and grid 2 . This is simply because of weaker resolved mixing which results in more data noise.

Fuel mass fraction results are displayed for experimental, RANS, multi-scale, and multi-scale with adaptive $S c_{t}$ approaches. The results are shown at two axial locations $x / D=5$ and $x / D=25$ in Figure 4 and Figure 5 respectively. At $x / D=5$, RANS results show two kidney vortices that are largely unmixed. When the multi-scale approach is used these two vortices are reduced in size and appear closer to each others in grid 1. In grid 2 these two vortices merge into one mixed core in the center similar to findings in the experimental measurements. The concentration of the fuel core is still over predicted in both grid 1 and grid 2 . When applying the adaptive $S c_{t}$ extension, results were largely unchanged. In the grid 1 the kidney vortices were closer and formed a single core. The contour also shows slightly larger attachment to the wall. For grid 2, only a slight reduction in core fuel concentration is observed. Overall, although insignificant, the results were closer to the experimental measurements. 


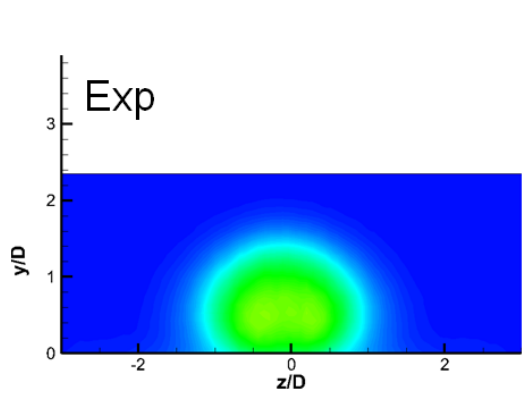

Multi-scale $\mathrm{Sc}_{\mathrm{t}}=0.7$

Multi-scale $S c_{t}=$ Var
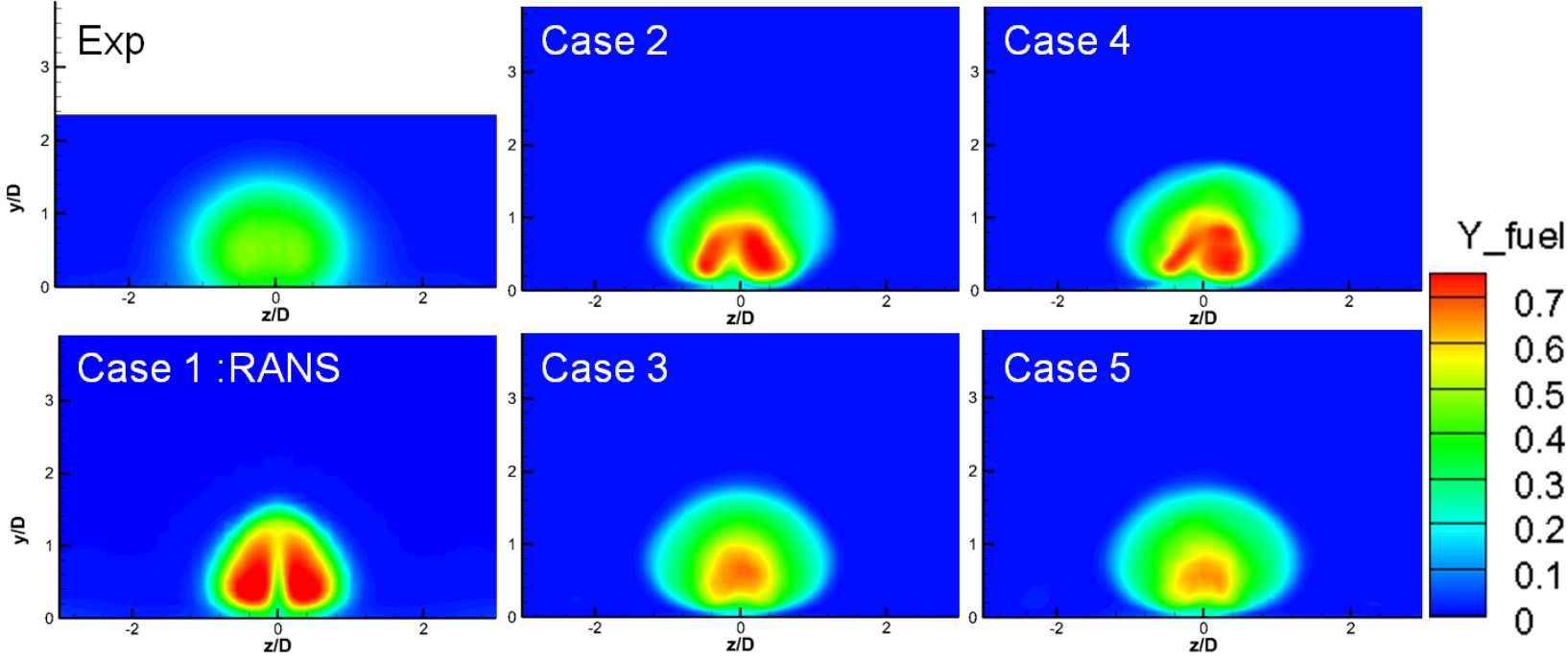

Figure 4. Fuel mass fraction contours at $x / D=5$ for experimental and different numerical approaches. In multi-scale simulations the results of grid 1 are on top (Cases 2 and 4) and grid 2 on the bottom (Cases 3 and 5).

At $x / D=25$, the RANS results show a lifted circular core that is attached to the wall through a slender neck of fuel. Multi-scale results at grid 1 show a more diffuse core with the fuel neck occurring closer to the wall. At grid 2, the results compare better to the experiment and show no fuel neck and a more diffuse fuel core. In all cases, there is a slight over prediction in the fuel penetration height. With the adaptive approach extension, grid 1 results were more diffuse and necking occurred closer to the wall than the constant $S c_{t}$ multi-scale. There was however, necking at the top of the fuel core on the left side that is asymmetric and is not predicted by the experiment. With grid 2, results improved slightly in resembling the experiment when using the adaptive $S c_{t}$ extension for grid 2.

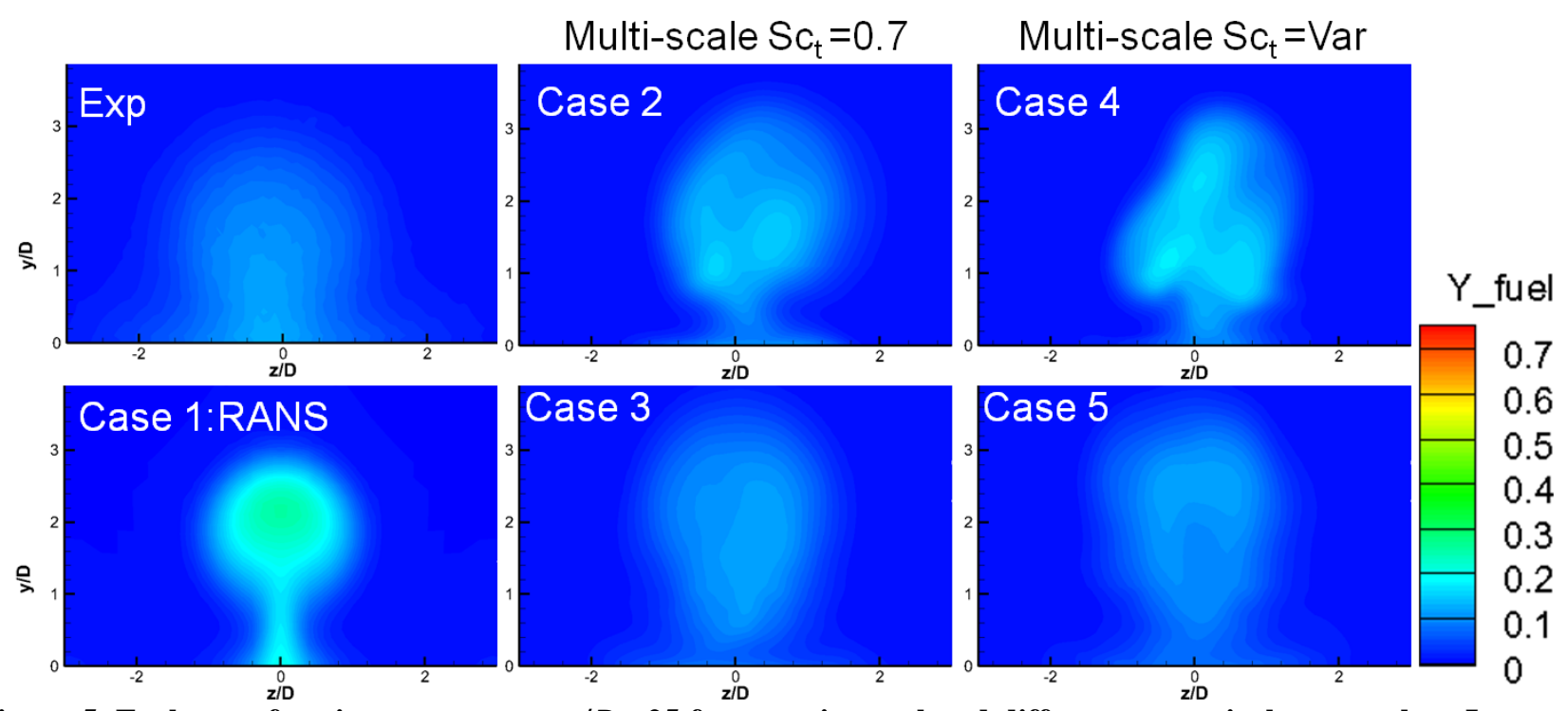

Figure 5. Fuel mass fraction contours at $x / D=25$ for experimental and different numerical approaches. In multi-scale simulations the results of grid 1 are on top (Cases 2 and 4 ) and grid 2 on the bottom (Cases 3 and 5).

B. Effect of numerical viscosity on turbulent diffusivity 
In the LES community, it is often argued that high-order-low-dissipation schemes are needed for an accurate LES simulation. This is because the error caused by the scheme often produces numerical viscosity that is on the order of eddy viscosity provided by the sub-grid model. In multi-scale modeling, numerical viscosity also plays an important role preventing the model from resolving smaller turbulent structures. In this effort, standard second order upwinding is used to model convective terms in the momentum equation leading to relatively high numerical dissipation. Therefore, finer grids are needed to produce the same size turbulent structures that are found in other researchers' results for the same problem ${ }^{17,20}$.

For multi-scale modeling, the amount of turbulent mass diffusivity is determined by the Reynolds diffusion term in the mass transport equation, namely, $D_{i_{m}}^{R} \approx-\frac{\mu_{t}}{S c_{t}} \frac{\partial \tilde{Y}_{m}}{\partial x_{j}}$. This term is directly proportional to the value of the momentum eddy viscosity $\left(\mu_{t}\right)$. In RANS modeling, this is acceptable because the value of the eddy viscosity is much larger than the numerical one. In multi-scale modeling, however the resolved eddy size is determined by the sum of all three viscosities. Dissipation in the momentum equation caused by the laminar, numerical and eddy viscosities determine the size of the resolved turbulent structures present in the solution. For example by not accounting for numerical diffusivity, the fuel diffuses in the sub-filter within eddies much smaller than those present in the solution (determined by the eddy viscosity alone). This causes limited turbulent mass diffusion in the overall solution.

One way to separate the effects of the numerical viscosity caused by the grid and the numerical scheme is to simply set the eddy viscosity to zero thus using no turbulence model. This method is equivalent to conducting an LES simulation with the sub-grid model implicitly defined as the numerical viscosity providing an upper limit to the turbulent oscillations in the solution for a particular grid and numerical scheme. This method was first named Monotone Integrated LES (MILES) by Boris ${ }^{34}$ and an extensive analysis can be found in the book by Grinstein et al. ${ }^{35}$.
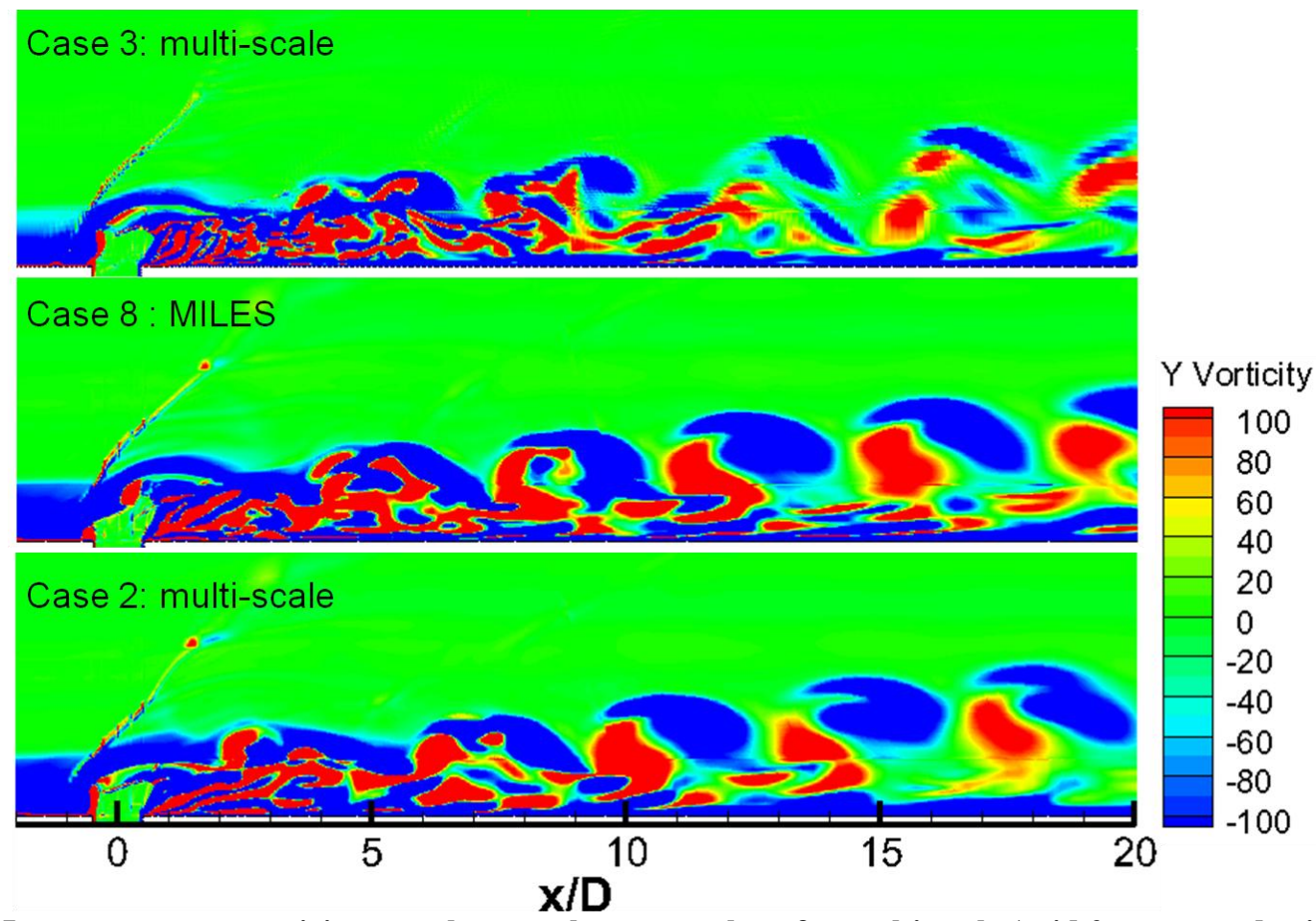

Y Vorticity

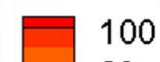

80

60

40

20

$-20$

$-40$

$-60$

$-80$

100

Figure 6. Instantaneous $y$ vorticity snapshots on the center plane for multi-scale (grid 2 on top and grid 1 on the bottom) and MILES (grid1) approaches. Maximum and minimum limited to 100, -100

Figure 6 shows the $y$ vorticity on the center plane for grid 1, and grid 2 (without adaptive $S c_{t}$ approach) as well as the MILES approach (zero eddy viscosity) for grid 1. This figure gives a qualitative assessment for the numerical viscosity. The vortices in the MILES solution are sized in between grid 1 and grid 2, but closer in size to the grid 1. This indicates that the eddy viscosity in grid 2 is less than the numerical viscosity in grid 1 . We could also say that 
the numerical viscosity is not orders of magnitude larger than the eddy viscosity in grid 1 because the MILES solution displays smaller vortices than those seen in grid 1 results.

Fuel mass fractions' contours for multi-scale grid 1and grid 2 and grid 1 MILES solution are displayed in Figure 7. It is seen that fuel in the MILES solution is much less diffusive than grid 1 and has about the same diffusivity as grid 2. This observation is in line with effect of the numerical viscosity on turbulent diffusion. Even though numerical viscosity has caused larger vortices in the MILES solution (slightly smaller than grid 1), the fuel was not dispersed in the same manner. In fact, fuel dispersion in the coarse MILES solution is comparable to grid 2.
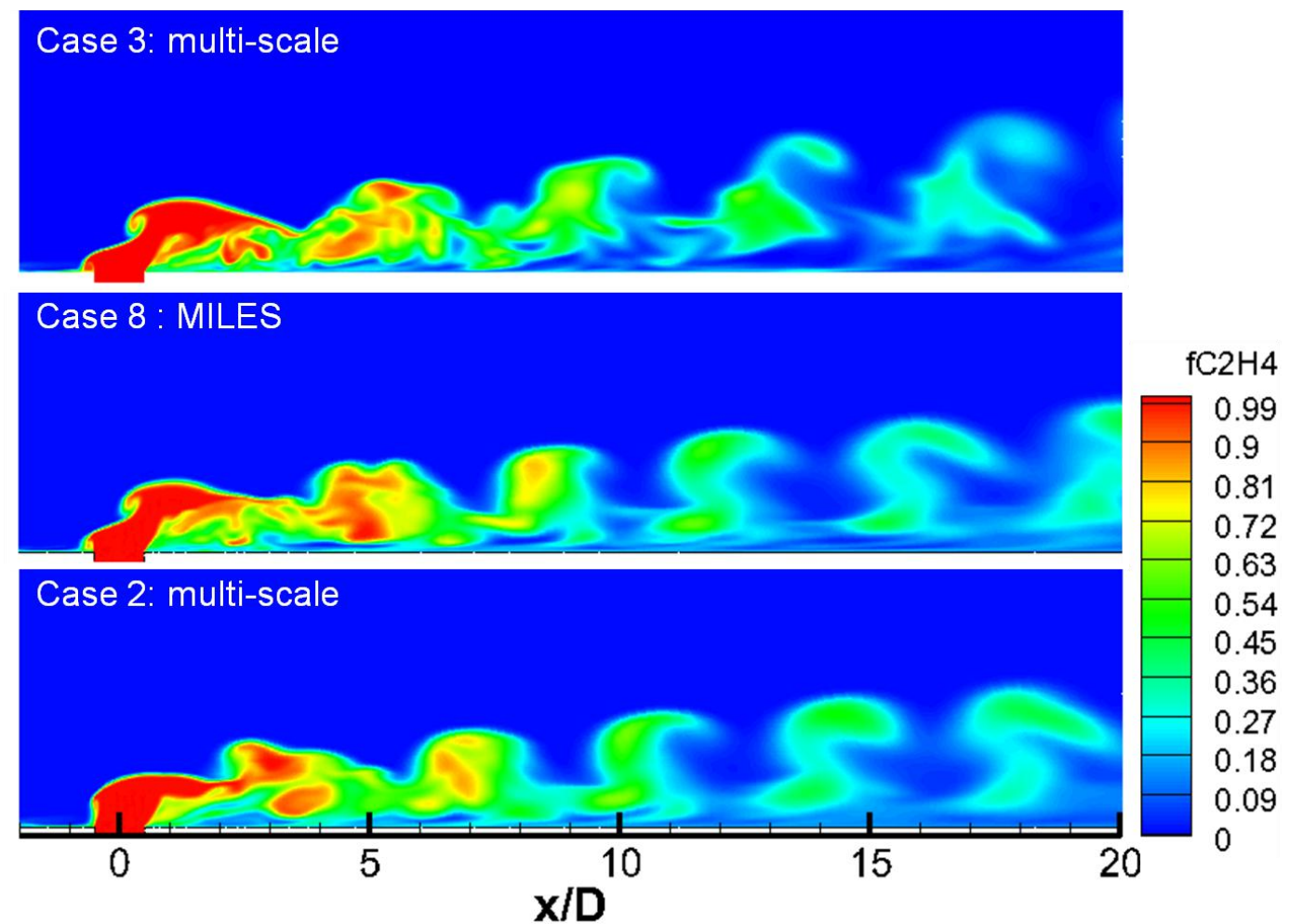

Figure 7. Instantaneous fuel mass fraction snapshots on the center plane for multi-scale (grid 2 on top and grid 1 on the bottom) and MILES (grid1) approaches.

To include the effect of numerical viscosity one must modify the Reynolds diffusion term as follows

$$
D_{i_{m}}^{R} \approx-\frac{\mu_{t}+\mu_{\text {num }}}{S c_{t}} \frac{\partial \tilde{Y}_{m}}{\partial x_{j}}
$$

It is difficult to calculate the numerical viscosity without a solution of higher order convection scheme which is not available and would make the computation impractical. One way to crudely estimate the numerical viscosity is to use the generalized Smagorinsky coefficients ${ }^{36-37}$ and estimate it as follows,

$$
\mu_{\mathrm{num}}=\bar{\rho} C_{\mathrm{gs}} L_{G}^{2}|S|
$$

The generalized coefficient, $C_{\mathrm{gs}}$, value suggested by Mossi et al. ${ }^{37}$ for a second order scheme in a compressible fully developed flow case is 0.2. This value is not universal and should be refined further for this particular case but we will use it here as a rough estimate for numerical viscosity. 
When the numerical viscosity is taken into account fuel mass fraction are calculated for grid 1 and grid 2 . The results are shown in Figure 8. Both the mixing and the fuel structure are in good agreement with the experiment. Grid 1 results have improved significantly especially in the near field showing more attachment to the wall and higher overall mixing consistent with the experiment. Grid 2 showed slight improvement over standard multi-scale at $x / D=5$ and better resemblance of the experiment at $x / D=25$. This is because near field grid resolution is so fine that diffusion within the sub-filter field becomes unimportant. Coarser grid in the far field, however, allows subfilter turbulent mass diffusion to play a larger role.

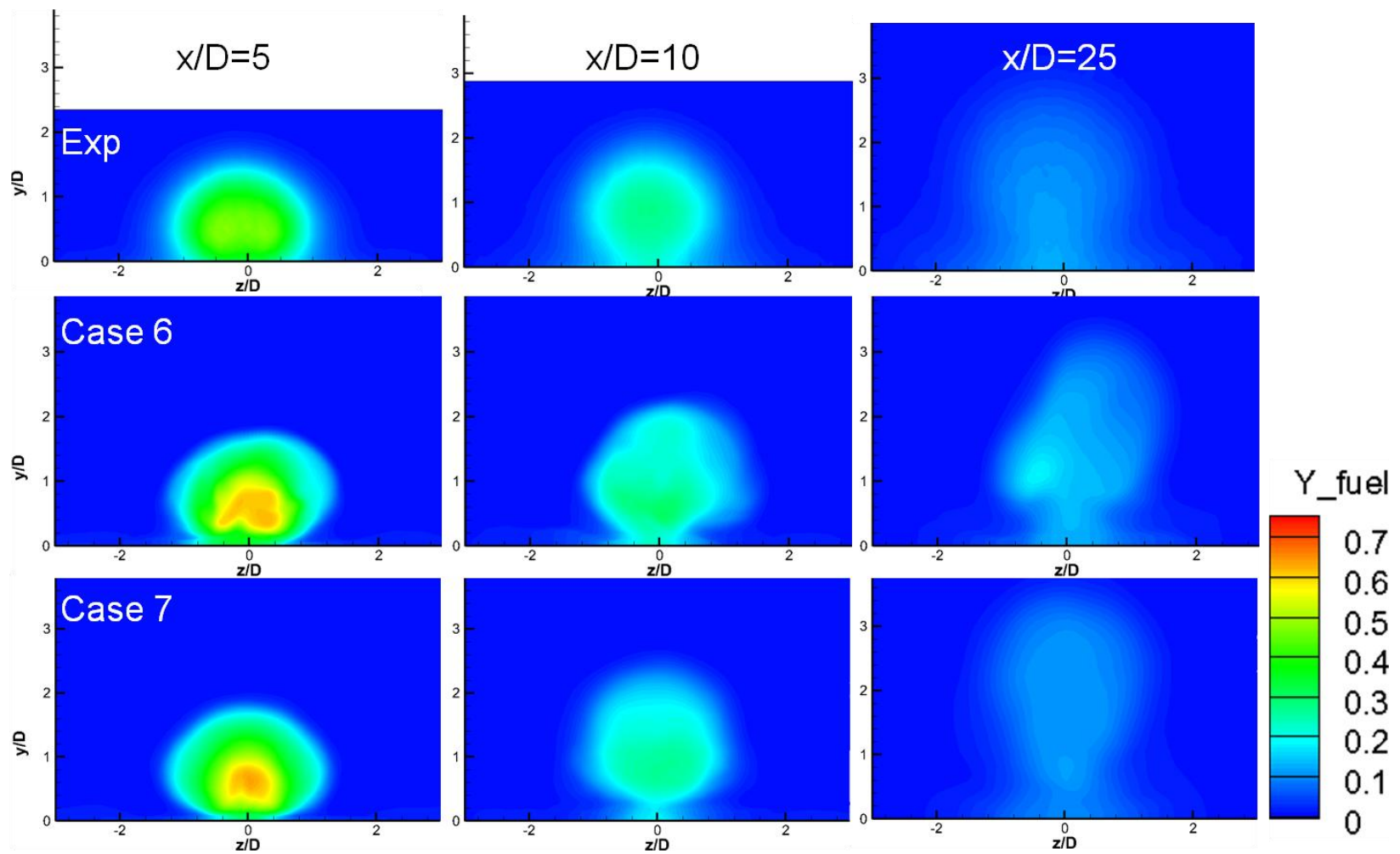

Figure 8. Fuel mass fraction predictions for the multi-scale adaptive $S c_{t}$ approach with numerical viscosity correction. Experimental measurements on the top, grid 1 in the middle (Case 2) and grid 2 on the bottom (Case 7)

\section{Adaptive turbulent Schmidt number effect on solution and mixing}

The difficulty in predicting experimental fuel distribution lies largely in the near field. Specifically, at $x / D=5$, fuel core concentrations were over predicted at varying degrees by all methods used in both current and previous efforts $^{19}$. It was also over predicted by others using hybrid RANS/LES ${ }^{17}$. This is mainly because near the fuel plum and the barrel shock, there is a high degree of unsteadiness that must be captured correctly. This unsteadiness works in mixing the fuel on the average sense in the near field region. Figure 9 shows average mass fraction iso-surfaces for cases 2, 3 and 6 as well as streamlines and bow shock pressure iso-surface for case 3 . The fuel coming from the center of the injector is projected along the top of the jet and is diffused conventionally as it travels downstream. Most of the unsteady mixing takes place in fuel carried by the horseshoe vortices which are originated from the edges of the injector. Those vortices are generated behind the injector in the area between the bow shock and its footprint on the boundary layer. Due to strong unsteadiness at the fuel plume near the barrel shock, the horseshoe vortices lose their strength on the average sense and diffuse with the main flow. The unsteady mixing process is stronger as we refine the grid causing the horseshoe vortices to diffuse faster.

Average fuel mass fractions are displayed as iso-surfaces in Figure 9 and as color contours on the center plane in Figure 10 cases 2, 3, and 6. As the grid is refined, fuel rich iso-surfaces become shorter as indicated in Figure 9 and the fuel vortex following the plume in Figure 10 becomes less intense due to unsteady mixing. It is the goal of this effort to model this mixing process in the near field using the adaptive $S c_{t}$ approach. It is therefore logical to use $S c_{t}$ based on the resolved field resulting in more mass diffusion in highly resolved regions. We can compare the fuel 
structure of case 6, which uses the adaptive $S c_{t}$ multi-scale approach with the numerical viscosity correction, to case 3 which is a simple refinement over case 2 using standard constant $S c_{t}$ multi-scale approach. In case 6, the fuel vortex is less intense and the fuel rich iso-surface is shorter resembling the mixing dynamics of the finer grid in case 3.

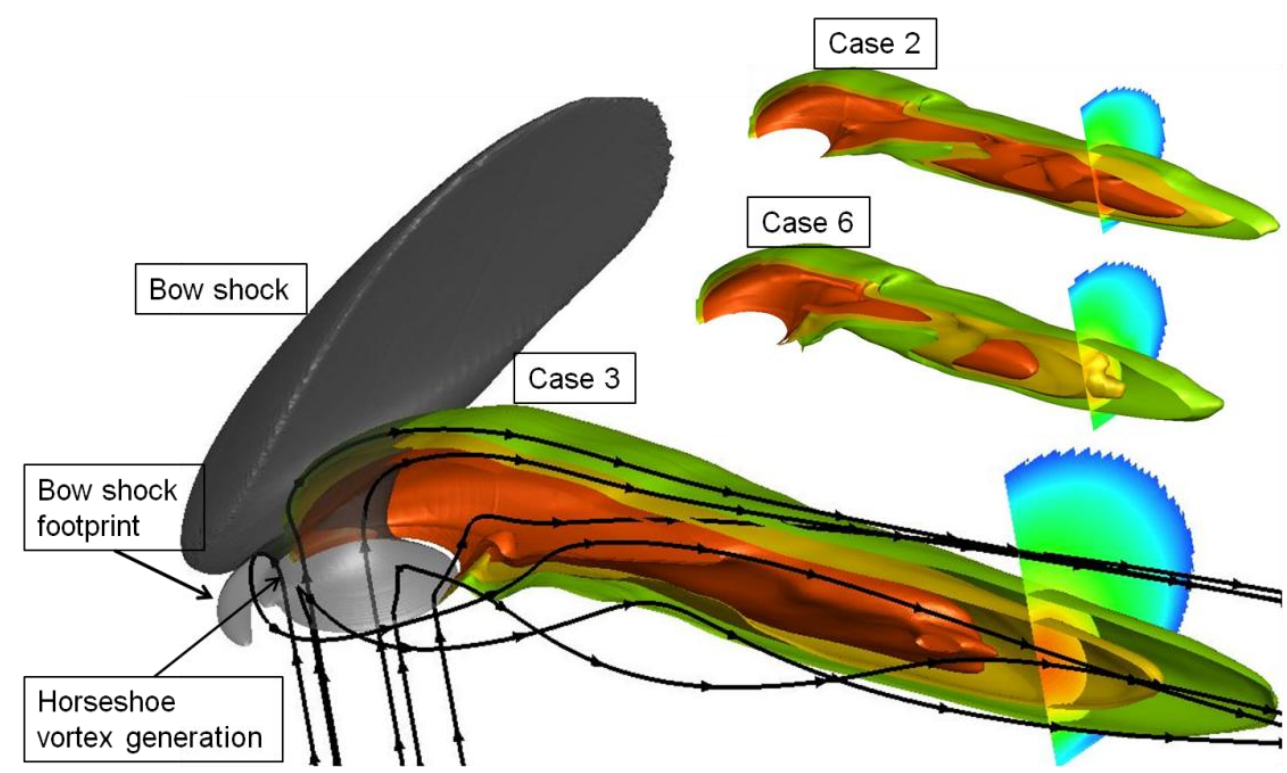

Figure 9. Average bow shock pressure iso-surface and fuel streamlines for case 3 (fine grid). Average fuel mass fractions iso-surfaces and $x / D=5$ slice for cases 2 (coarse grid), 3 (fine grid), and 6 (coarse grid).

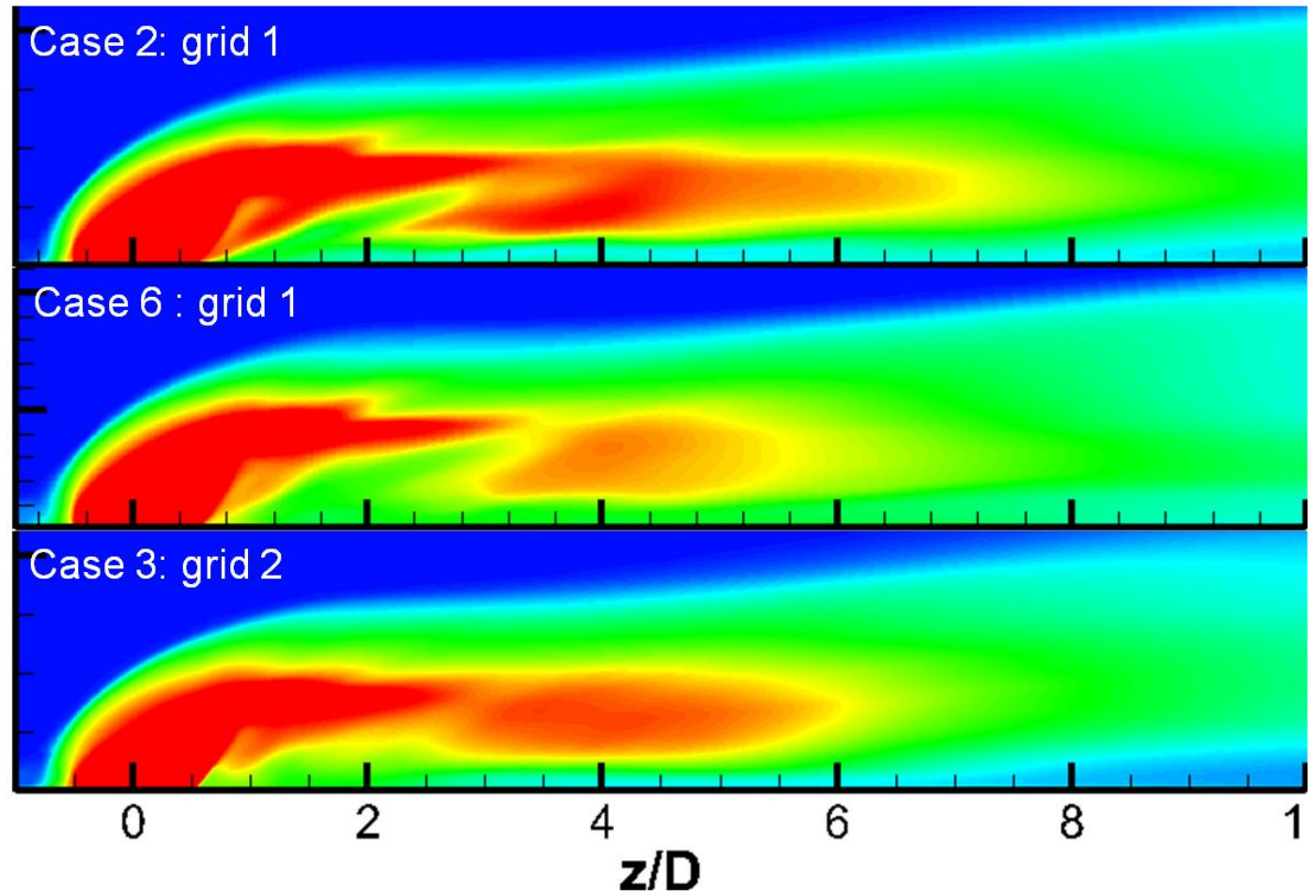

Figure 10. Average fuel mass fractions at center plane for cases 2 ( coarse grid), 3 (fine grid), and 6 (coarse grid).

Figure 11 displays average pressure contours on the center plane for cases 3 and 6 . The pressure contours are similar showing the bow the barrel shock near the injector. It is evident that the adaptive Sct multi-scale approach 
had no adverse effects on the pressure field. Figure 12 shows pressure contours on the bottom wall for cases 3, 6 as well as experimental pressure sensitive paint (PSP) measurements that were conducted recently ${ }^{38}$ at Air Force Research lab using fast response paint and high speed cameras. Both computed results (cases 3 and 6) are in good agreement with experimental measurements and each others. Pressure contours show foot prints of the bow and barrel shocks that are indistinguishable from each others in experimental PSP but can be clearly defined on the fine grid in case 3. The wake region downstream is in better agreement with the experimental measurements in case 3 , however, differences are not significant and could be accounted to the refinement of the grid.
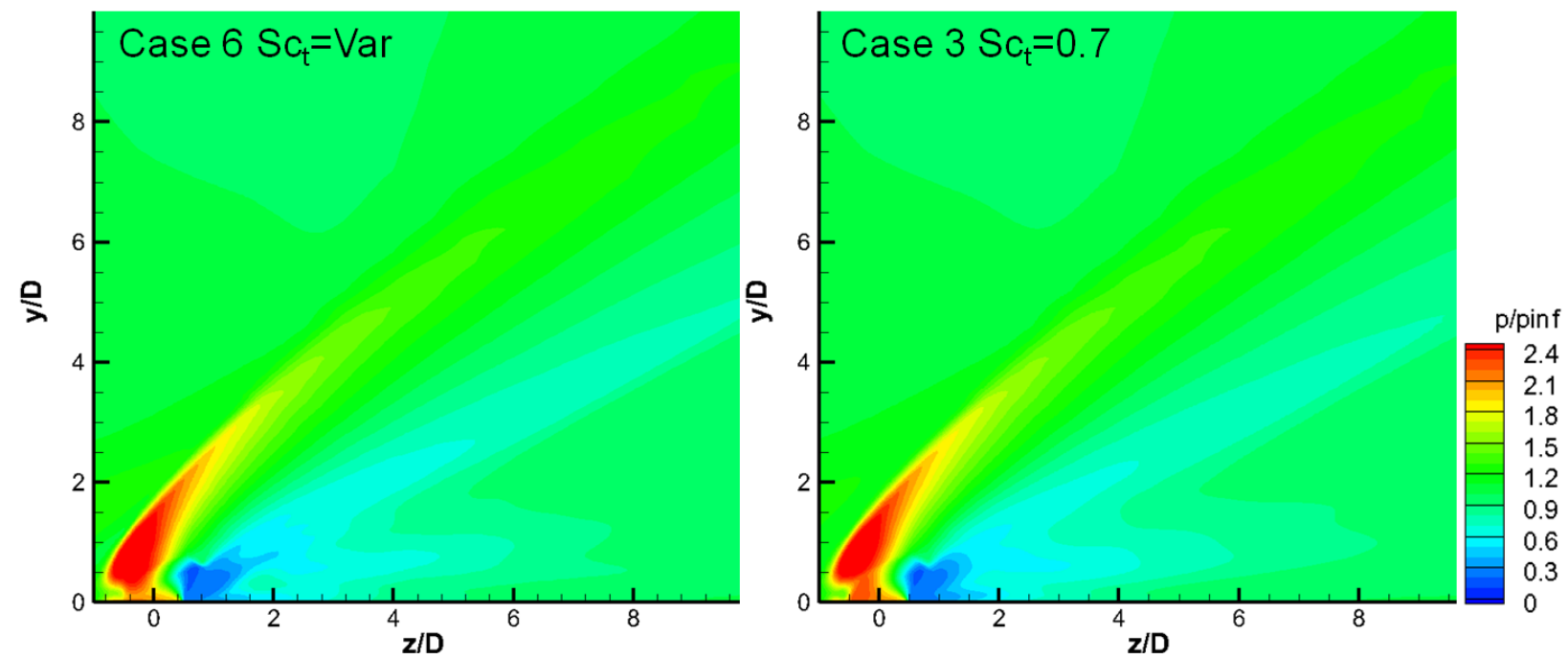

Figure 11. Center plane pressure contours for cases 3 (fine grid) and 6 (coarse grid). Injector is located at $z / D=0$.
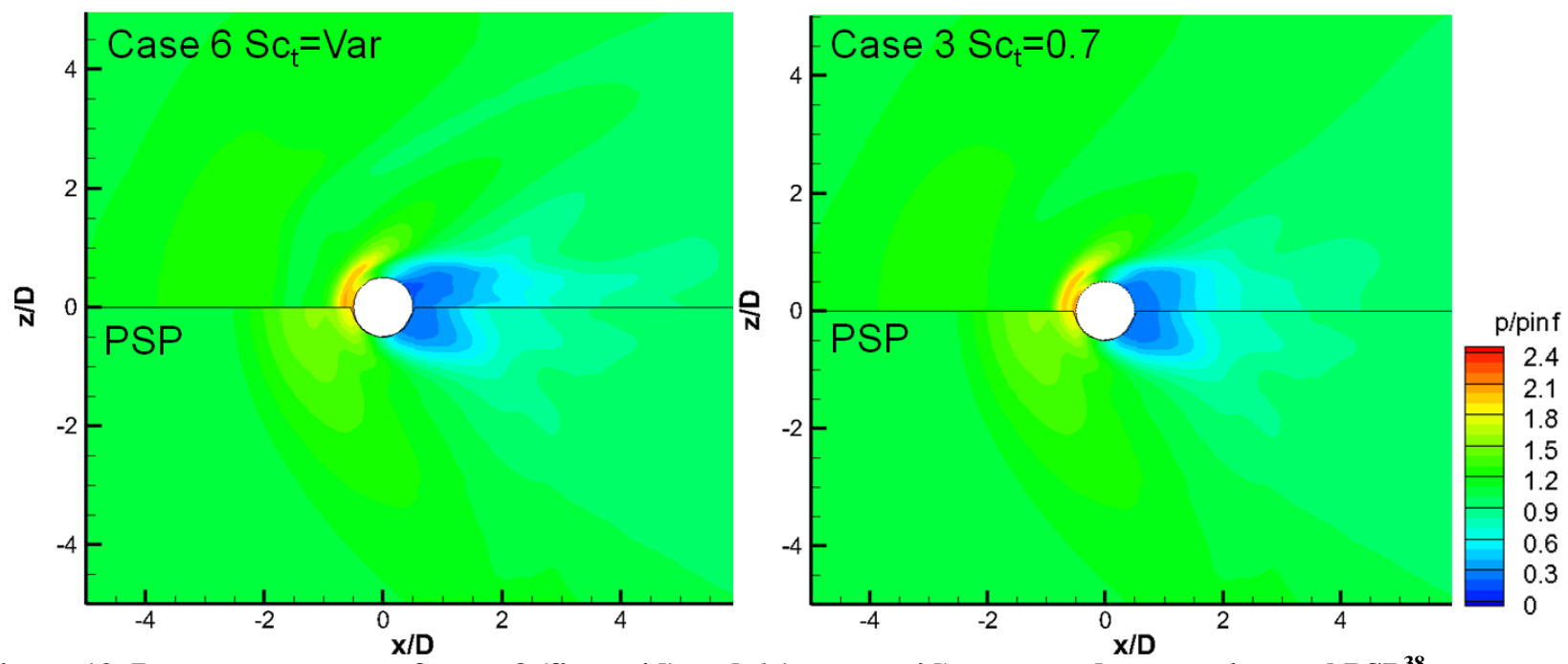

Figure 12. Pressure contours of cases 3 (fine grid) and 6 (coarse grid) compared to experimental PSP ${ }^{38}$.

\section{Summary and Conclusions}

An adaptive Schmidt number extension to the multi-scale model was successfully developed and implemented. As the simulation proceeds, average resolved turbulent mass and momentum viscosities are calculated and a turbulent Schmidt number is defined based on their ratio. When applied directly into the RANS sub-filter model, the solution showed fuel structure closer to the experimental measurements however, changes over the constant approach were not significant. When the numerical viscosity is taken into account to calculate turbulent mass diffusivity, the solution improves significantly to be in a good agreement with experimental measurements. 
Turbulent Schmidt number contours showed more symmetry as the grid was refined because resolved data becomes more dominant than noise resulting from the averaging procedure. This shows that the approach should be used only on grids producing adequate level of resolved turbulent fluctuations. A grid too fine, however, would not benefit much from the adaptive approach, since most of the mass diffusion will be occurring in the resolved field. Because of the use of highly dissipative second order upwind for the convection terms, better estimates for the numerical viscosity should be obtained rather than the crude estimate used in this efforts. Higher order schemes should also be considered in the multi-scale modeling framework to minimize the effect of numerical viscosity. Since only one experimental case is investigated, more cases should also be conducted to show the validation and applicability of the approach proposed in this effort.

\section{Acknowledgements}

Ez A Hassan would like to acknowledge the SMART program for their support. Portions of the computations were performed on AFRL high performance computing clusters.

\section{References}

1. Tam, C.-J., Baurle, R. A., and Gruber, M. R. "Numerical Study of Jet Injection into a Supersonic Crossflow," 35th AIAA/ASME/SAE/ASEE Joint Propulsion Conference and Exhibit. Vol. A99-31100, Los Angeles, california, 1999.

2. Menter, F. R. "Zonal two equations k-w turbulence models for aerodynamic flows," AIAA Vol. 93, No. $2909,1993$.

3. Wilcox, D. C. "Simulation of Transition With a Two-Equation Turbulence Model," AIAA Journal Vol. 32, 1994, pp. 247-254.

4. Gruber, M. R., Nejad, A S., J. C. Dutton. "An Experimental Investigation of Transverse Injection from Circular and Elliptical Nozzles into supersonic Crossflow," Wright Lab Technical Report Vol. WL-TR-96-2102, 1996.

5. Gruber, M. R., Nejad, A. S., Chen, T. H., and Dutton, J. C. "Large structure convection velocity measurements in compressible transverse injection flowfields," Experiments in Fluids Vol. 22 (1997), 1997, pp. 397-407.

6. Gruber, M. R., Nejad, A. S., Chen, T. H., and Dutton, J. C. "Compressibility Effects in Supersonic Transverse Injection Flowfields," Physics of Fluids Vol. 9, No. 5, 1997, pp. 1448-1461.

7. Palekar, A., Truman, C. R., and Vorobieff, P. "Prediction of Transverse Injection of a Sonic Jet in a Supersonic Crossflow," 36th AIAA Plasmadynamics on Lasers Conference. Vol. 2005-5366, Toronto, Ontario Canada, 2005.

8. Maddalena, L., Campioli, T. L., and Schetz, J. A. "Experimental and Computational Investigations of Light-Gas Injectors in Mach 4.0 Crossflow," Journal of Propulsion and Power Vol. 22, No. 5, 2006.

9. Nichols, R. H., and Tramel, R. "Applications of a Highlt Efficient Numerical Method for Overset Mesh Moving Body Problems," AIAA Vol. 97-2255, 1997.

10. Sinha, N., Dash, S., and Chidambaram, N. "A perspective on the Simulation of Cavity Aeroacoustics," AIAA Vol. 980286, 1998.

11. Spalart, P., Jou, W., and Strelets, M. Comments on the Feasibility of LES for Wings and ona Hybrid RANS/LES Approach: Greyden Press, Columbus, OH, 1997.

12. Kawai, S., and Lele, S. K. "Large-Eddy Simulation of Jet Mixing in Supersonic Crossflows," AIAA Journal Vol. 48, No. 9, 2010, pp. 2063-2083.

doi: 10.2514/1.J050282

13. Santiago, J. G., and Dutton, J. C. "Velocity Measurements of a Jet Injected into a Supersonic Crossflow," Journal of Propulsion and Power Vol. 11, No. 2, 1997, pp. 315-323.

doi: $10.2514 / 2.5357$

14. Peterson, D. M., Subbareddy, P. K., and Candler, G. V. "Assessment of Synthetic Inflow Generation for Simulating Injection into Supersonic Crossflow," 14th AIAA/AHI Space Planes and Hypersonic Systems and Technologies Conference. 2006.

15. Boles, J. A. "Hybrid Large-Eddy Simulation/Reynolds-Averaged Navier-Stokes Methods and Predictions for Various High-Speed Flows," Aerospace Engineering. Vol. Doctor of Philosophy, North Carolina State University, Raleigh, NC, 2009.

16. Boles, J. A., and Edwards, J. R. "Hybrid LES/RANS Simulation of Transverse Sonic Injection into a Mach 2 Flow," 46th AIAA Aerospace Sciences Meeting and Exhibit. Vol. 2008-622, Reno, Nevada, 2008.

17. Boles, J. A., Edwards, J. R., and Baurle, R. A. "Large-Eddy/Reynolds-Averaged Navier-Stokes Simulations of Sonic Injection into Mach 2 Crossflow," AIAA Journal Vol. 48, No. 7, 2010, pp. 1444-1456.

doi: 10.2514/1.J050066

18. Lin, K.-C., Ryan, M., Carter, C., Gruber, M., and Raffoul, C. "Scalability of Ethylene Gaseous Jets for Fueling HighSpeed Air-Breathing Combustors," 47th AIAA Aerospace Sciences Meeting and Exhibit. Vol. 2009-1423, Orlando, Florida, 2009. 
19. Hassan, E., Aono, H., Boles, J., Davis, D., and Shyy, W. "Multi-Scale Turbulence Model in Simulation of Supersonic Crossflow," 49th AIAA Aerospace Sciences Meeting including the New Horizon Forum and Aerospace Exposition Orlando, Florida, 2011.

20. Peterson, D. M., and Candler, G. V. "Supersonic Combustor Fuel Injection Simulation Using a Hybrid RANS/LES Approach," 48th AIAA Aerospace Sciences Meeting Including the New Horizon Forum and Aerospace Exposition. Orlando, Florida, 2010.

21. Reynolds, A. J. "The Prediction of Turbulent Prandtl and Schmidt Numbers," Int. J. Heat and Mass Transfer Vol. 18, 1975, pp. 1055-1069.

22. Negano, Y., Kondoh, M., and M.Shimada. "Multiple time-scale turbulence model for wall and homogenous shear flows based on direct numerical simulation," Int. J. Heat Fluid Flow Vol. 18, No. 4, 1997, pp. 347-359.

23. Sommer, T. P., So, R. M. C., and Zhang, H. S. "Near-wall variable Prandtl-Number turbulence model for compressible flows," AIAA Journal Vol. 31, No. 1, 1993, pp. 27-35.

24. Guo, Y., He, G., Hsu, A. T., Brankovic, A., Syed, S., and Liu, N. S. "The Development of a Variable Schmidt Number Model for Jet-in-Crossflow Using Genetic Algorithms," AIAA Paper, No. 99-0671, 1999.

25. Kenzakowski, D. C., Papp, J., and Dash, S. M. "Evaluation of Advanced Turbulence Models and Variable Prandtl/Schmidt Number Methodology for Propulsive Flows," AIAA Paper, No. 2000-0885, 2000.

26. Brinckman, K. W., Kenzakowsi, D. C., and Dash, S. M. "Progress in Practical scalar Fluctiation Modeling for High Speed Aeropropulsive Flows," AIAA Paper, No. 2005-0508, 2005.

27. Keislter, P. G. "A Variable Turbulent Prandtl and Schmidt Number Model Study for Scramjet Applications," Mechanical and Aerospace Engineering. Vol. PhD, North Carolina State University, Raleigh, North Carolina, 2009.

28. Luke, E. A., and Cinnella, P. "Numerical simulations of mixtures of fluids using upwind algorithms," Computers \& Fluids Vol. 10, 2007, pp. 1547-1566.

29. Luke, E. A., and George, T. "Loci: A rule-based framework for parallel multidisciplinary simulation synthesis," Journal of Functional Programming Vol. 15, No. 3, 2005, pp. 477-502.

30. Roe, P. L. "Approximate Riemann solvers parameter vectors and difference schemes," Journal of Computational Physics Vol. 43, 1981, pp. 357-372.

31. Germano, M. "From RANS to DNS: Towards a Bridging Model," ERCOFTAC Series Vol. 7, 1999, pp. $225-236$.

32. Johansen, S. T., Wu, J., and Shyy, W. "Filter-based unsteady RANS computations," International Journal of Heat and Fluid Flow Vol. 25(2004), 2004, pp. 10-21.

33. Nichols, R. H., and Nelson, C. C. "Application of Hybrid RANS/LES Turbulence Models," 41st Aerospace Science Meeting and Exhibit. Reno, Nevada, 2003.

34. Boris, J. P. "On Large Eddy Simulation Using Subgrid Turbulence Models," in Whither Turbulence? Turbulence At The Crossroads, Lecture Notes in Physics Vol. 357, 1990, pp. 344-353.

35. Grinstein, F. F., Margolin, L. G., and Rider, W. J. Implicit Large Eddy Simulation: Computing Turbulent Fluid Dynamics: Cambridge University Press, 2007.

36. Garnier, E., Mossi, M., Sagaut, P., Comte, P., and Deville, M. "On the Use of Shock-Capturing Schemes for LargeEddy Simulation," Journal of Computational Physics Vol. 153, No. 2, 1999, pp. 273-311.

doi: $10.1006 /$ jcph. 1999.6268

37. Mossi, M., and Sagaut, P. "Numerical investigation of fully developed channel flow using shock-capturing schemes," Computers \& Fluids Vol. 32, No. 2, 2003, pp. 249-274.

38. Crafton, J., Forlines, A., Palluconi, S., Hsu, M., Carter, C., and Gruber, M. "Investigation of Transverse Jet Injections in a Supersonic Crossflow Using Fast Responding Pressure-Sensitive Paint," AIAA Vol. submitted, 2011. 